\title{
Experimental Assessment of Fouling Effects in a Multistage Axial Compressor
}

\author{
Nicola Casari, Michele Pinelli, Pier Ruggero Spina, Alessio Suman* and Alessandro Vulpio \\ University of Ferrara - Department of Engineering, via Saragat, 1, 44122 Ferrara, Italy
}

\begin{abstract}
The study of the adhesion of micro sized particles to gas turbine internal surfaces, commonly known as gas turbine fouling, has gained increasing attention in the last years due to its dramatic effect on machine performance and reliability. On-field fouling analysis is mostly related to visual inspections during overhaul and/or programmed stops, which are performed, in particular, when gas turbine performance degradation falls under predetermined thresholds. However, these analyses, even if performed in the most complete as possible way, are rarely (or never) related to the conditions under which the gas turbine contamination takes place since the affecting parameters are difficult or even impossible to be adequately monitored. In the present work, a small scale multistage axial compressor is used to experimentally simulate the fouling phenomenon. The test rig allows the accurate control of the most relevant operating parameters which influence the fouling phenomenon. The compressor performance loss due to particle contamination has been quantitatively assessed. Soot particles appear stickier, especially in the presence of high humidity, and represent the most harmful operating conditions for the compressor unit. The deposits on the stator vanes and the rotor blades have been detected and post-processed, highlighting the most affected regions of each compressor stage employing an image analysis package tool.
\end{abstract}

\section{Introduction}

The adhesion of micro-sized particles is the main responsible for gas turbine fouling [1]. Such adhesion generates a deposits build-up on the internal surfaces of the power units, in both cold and hot sections [2 - 4]. Operating conditions as machine load and pollution speed-up the detrimental effects and the progression of the performance loss. The concentration of natural and/or artificial micro-sized particles, together with the air humidity, represents the drivers of the fouling issues [5]. Due to this, power units are affected by deposits that develop over time, reducing the operability of the engine $[6,7]$. On-field detections have revealed that the fouling issue affects the unit performance despite the presence of filtration systems [8] and, only with a periodic (frequent) online washing operation, the reliability of the engine remain acceptable. During the machine overhaul, inspections of the deposits are rarely reported [1,9-11], and, when provided, the details useful for relating the pattern to the operating conditions of the units are missed.

\footnotetext{
* Corresponding author: alessio.suman@unife.it
} 
State this, only a few information can be drawn concerning the interaction between micro-sized particles and a multistage axial compressor. Finer particles appear to the most detrimental for the unit performance, and by introducing more effective filtration systems, the result is a lowered performance drop. Non-linear effects could be due to the modification of the compressor operating point, and, for particular regime operation, the detrimental action of the fouling on the pressure ratio could be masked by the variation of the flow coefficient $[12,13]$. As reported in $[5,11,14]$, front stages are more prone to collect particles, especially in the presence of high air humidity values and, washing operations remove the deposits from the front stages but promoting a re-deposit phenomenon in the rear ones.

This work has the aim to define a complete experimental assessment of the fouling issue on a multistage axial compressor. Compressor performance, particle sticking behaviour, and deposits patterns are detected after a specific test during which the compressor unit, starting from the clean condition, was fouled by micro-sized particles. The experimental investigation comprises the ingestion of soil and soot, under high relative humidity value at the compressor inlet. The data post-process is composed of three main parts: (i) the calculation of the capture efficiency of the whole machine, carried out with a laser spectrometer at the compressor outlet, (ii) the overtime variation of the pressure ratio during the exposure time, and (iii) the photographic report of the entire compressor flow path performed, after each text, using a visual inspection of the entire unit coupled with a systematic image post process.

\section{Experimental apparatus}

The present experimental apparatus has the aim to assess the effects of compressor fouling in terms of (i) performance losses and (ii) deposits pattern. Testing the multistage compressor under controlled conditions, as reported in the previous section, gives the possibility to link the deposition phenomenon (collection efficiency and deposits pattern over the compressor flow path) to the compressor operation, precisely.

The test bench mounts the compressor of the engine Allison $250 \mathrm{C} 18$. For this engine, the multistage axial unit is coupled with a centrifugal stage for an overall pressure ratio of 6.2 and a mass flow rate of $1.36 \mathrm{~kg} / \mathrm{s}$ at the design speed of 51,600 rpm [15]. An electric motor drives the unit and it is installed on a pipeline equipped with several devices for studying the fouling phenomenon. In particular, the experimental apparatus depicted in Fig. 1, comprises the following devices:

- an inlet air treatment section able to filter the inlet air (by EPA09-class filters) and to increase and control the humidity value by a steam injector driven by a steam generator;

- a contamination section that provides the injection of the contaminants in the airflow. In this section, the particle feeder TOPAS SAG40U prepares and injects the contaminants by a two Venturi eductors which guarantee the proper particle injection process;

- a dust monitoring system at the compressor outlet, equipped with the optical spectrometer TSI Particle Spectrometer OPS 3330 able to detect particles smaller than $10 \mu \mathrm{m}$;

- pressure transducers for monitoring the compressor performance;

- camera and light setup for detecting the deposits on the blade and vanes surfaces.

The dosing system comprises a rotating ring that is loaded by a rotating conveyor continuously. The particles, deposited onto the rotating rig, was picked up by the suction action provided by the first Venturi nozzle, driven by dry shop air. Therefore, in this first stage, the powder is prepared, deagglomerated, and dosed to the second Venturi eductor, mounted inside the compressor inlet pipeline. This second nozzle, driven by dry shop air, is responsible for the final powder deagglomeration and dispersion in the airflow stream at the compressor inlet section. The injection point is $2 \mathrm{~m}$-far from the inlet section allowing the 
kinematic equilibrium between powder and airflow as well as the uniform particle distribution at the compressor inlet section.

The particle monitoring system installed at the compressor outlet section provides the online measurement of the contaminant concentration in the airflow stream. The system collects a small portion of contaminated air, and by the light scattering technique, gives the particle count continuously. According to this, it is mandatory to ensure the isokinetic condition between the velocity at the inlet of the spectrometer probe and the velocity of the undisturbed flow, for avoiding the under/over-estimation of the particle concentration [16]. Given this, it is possible to calculate the stuck particles inside the compressor (i.e., the capture efficiency) since the particle feeder provides a constant and know airflow contamination. The assessment of the fouling effects is carried out by the evaluation of the compressor performance drop and the deposit patterns on the blade/vane surfaces. Compressor performance was monitored imposing (employing the electric motor governor) a fixed value for the correct rotational speed of 20,000 rpm and collecting the pressure at the compressor inlet and outlet sections. A careful evaluation of the measurement uncertainty was carried out based on the procedure reported in the standard ISO/IEC Guide 98-3:2008 [17] and EA-4/02M [18]. The compressor performance is taken by the evaluation of the pressure ratio $\beta$ of the multistage axial unit as

$$
\beta=\frac{p_{s, b l e e d}}{p_{s, \text { inlet }}}
$$

with its associated uncertainty expressed according to the uncertainty propagation theory

$$
\delta \beta=\sqrt{\left(\frac{\partial \beta}{\partial p_{s, \text { bleed }}}\right)^{2} \delta p_{s, \text { bleed }}^{2}+\left(\frac{\partial \beta}{\partial p_{s, \text { inlet }}}\right)^{2} \delta p_{s, \text { inlet }}^{2}+2 r\left(\frac{\partial \beta}{\partial p_{s, \text { bleed }}}\right)\left(\frac{\partial \beta}{\partial p_{s, \text { inlet }}}\right) \delta p_{s, \text { bleed }} \delta p_{s, \text { inlet }}}
$$

where the uncertainty of pressure values measured at the suction $\left(\delta \mathrm{p}_{\mathrm{s} \text {,inlet }}\right)$ and bleed $\left(\delta \mathrm{p}_{\mathrm{s}, \mathrm{bleed}}\right)$ sections are evaluated considering three different sources: (i) the uncertainty of the primary laboratory standard (PLS) device used for calibrating the pressure transducers at inlet and outlet compressor section $\left(U_{\mathrm{PLS}}\right)$, (ii) the residual uncertainty of the measurement chain $E_{\mathrm{R}}$ estimated by the difference between the reference value (provided by the PLS) and the average value of the signal provided by the measurement chain reordered for $2 \mathrm{~s}$

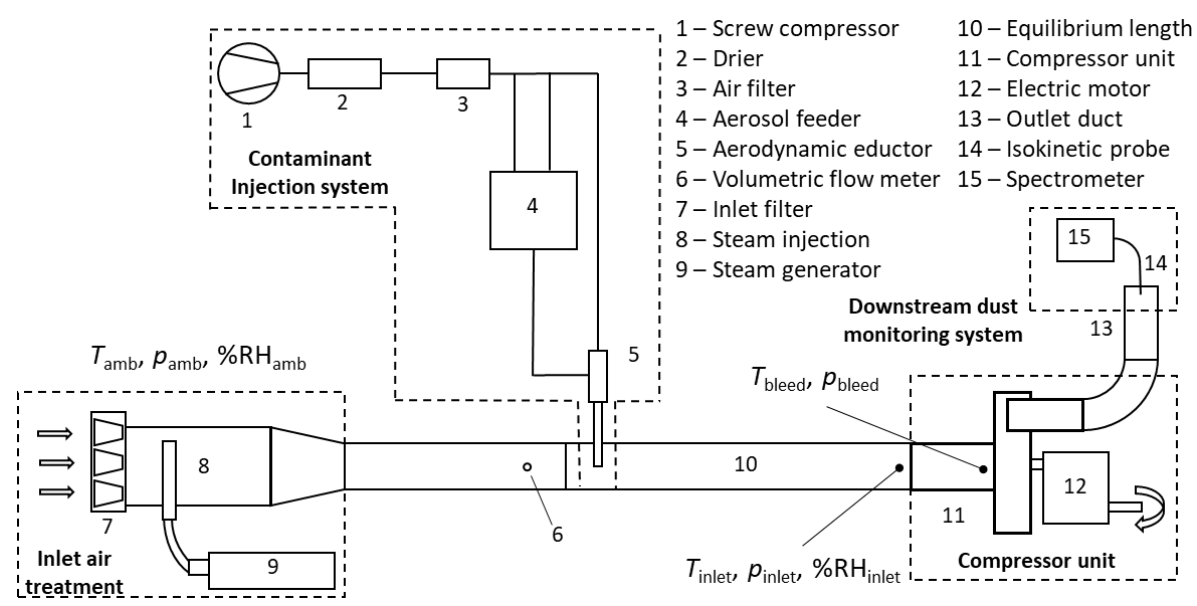

Fig. 1. Experimental apparatus for the experimental assessment of compressor fouling: block diagram and laboratory facility equipped with multiple cameras and light setup 
under a constant pressure input, and (iii) the Type A uncertainty related to the pressure measurement chain $U_{\sigma}$ is estimated by the acquisition of 2000 pressure points during a constant pressure input. Therefore, the final assessment of the uncertainty as

$$
\delta p=\sqrt{\left(\frac{U_{P L S}}{2}\right)^{2}+\left(\frac{E_{R}}{\sqrt{3}}\right)^{2}+\left(\frac{U_{\sigma}}{2}\right)^{2}}
$$

where the denominators refer to the uncertainty distribution for given uncertainty source. Gaussian probability distribution implies the division by 2 (adopted for $U_{\mathrm{PLS}}$ and $U_{\sigma}$ ) while the rectangular distribution adopted for the residual error $\left(E_{\mathrm{R}}\right)$ implies the division by $3^{1 / 2}$. Table 1 reports the uncertainty values for both measurement sections.

Finally, particular attention was put on the camera and light setup. Together with the compressor performance, the detection of the deposit patterns is necessary for the overall assessment of the fouling issue. For this reason, a specific setup is adopted for the present work. The compressor was dissembled, allowing the detection of the patterns over the stages. Thanks to this, the half compressor case (stator) was positioned on a realized-onpurpose holder while the rotor was kept on the original position. The deposits patterns are taken directly from the lab-testing facility employing dedicated cameras (Canon EOS M6 mirrorless digital camera with Tokina $100 \mathrm{~mm}$ lens with a resolution of 3552 x 2664 pixels) positioned to detect the entire flow path of the axial compressor divided according to the rotor and stator components. The white light setup is adopted for both parts of the compressor flow path with the intensity of $4000 \mathrm{~K}$. The detection procedure was carried out, ensuring the same camera position and the same light setup. Starting from these detections, the determination of the deposit pattern has been carried out through image processing. The open-source image processing package named IMAGE J [19], developed by the U.S. National Institutes of Health was used to analyze the digital pictures. Using the same procedure of [20], a macro has been used to subtract the picture of the clean stator and rotor to the picture of the dirt one and to convert the resultant image into a binary format. Finally, the picture is converted to show the deposits as the grayscale colored pattern on the picture. As a direct consequence of this, the rotor pattern analysis is more affected by a sort of noise due to the thermal expansion of the compressor during the test. This implies that the picture capture in the fouled condition appears slightly shifted from the clean one. By a dedicated analysis, this deviation is estimated equal to $0.5 \mathrm{~mm}$ at it is responsible for the post-process inaccuracies at the blade boundary in the case of rotor blade.

\section{Compressor fouling investigation}

The present investigation has the goal to show a comprehensive experimental assessment of the fouling phenomenon in a multistage axial compressor. Due to this, two different contaminants have been selected: a common soil powder, and a soot simulant. The first one is the standard powder known as Arizona Road Dust (ARD), while the second one is a paracrystalline carbon produced by the incomplete combustion of hydrocarbon and heavy petroleum products known as a Carbon Black (CB).

Table 1. Extended uncertainty values $(2 \sigma)$ for the pressure measurement at inlet and bleed sections

\begin{tabular}{|c|c|c|c|}
\hline Section & $\boldsymbol{U}_{\text {PLS }}[\mathbf{P a}]$ & $\boldsymbol{E}_{\mathbf{R}}[\mathbf{P a}]$ & $\boldsymbol{U}_{\boldsymbol{\sigma}}[\mathbf{P a}]$ \\
\hline Inlet & 50 & 330 & 66 \\
\hline Bleed & 56 & 420 & 76 \\
\hline
\end{tabular}


Since the fouling which affects land-based power units is mainly due to the micrometric particles, the powder selection has been focused on finer contaminants. Figure 2 shows the diameter distribution for the two selected powders as well as the statistics related to the average diameter $\left(d_{\text {mean }}\right)$ and the different classes of size $\left(d_{10}, d_{50}\right.$, and $\left.d_{90}\right)$. The powder samples were provided by Powder Technology Inc. according to the standard ISO 12103:2016 [21]. The number of injected particles per unit of time was kept constant (336 million particles per second) for all the conditions tested. Before being injected, the powder has been baked for $12 \mathrm{~h}$ at $70{ }^{\circ} \mathrm{C}$ to eliminate the moisture.

Thanks to the installation of the steam humidifier, the deposition tests have been carried out increasing the relative humidity values up to $80 \% \mathrm{RH}$. This value is kept constant during the exposure time, avoiding the condensation phenomenon at the compressor inlet section. As reported by [14] the condensation phenomenon could affect the inlet section and the first stage due to the local acceleration and pressure losses, varying the interaction between particles and blade surface and in turn the adhesion patterns.

Regarding the compressor operating conditions, a representative condition has been assumed for all the tests. The tests have been carried out at the quasi-idle condition at $20,000 \mathrm{rpm}$ as the corrected rotating speed [15]. In this operating condition, the compressor swallows $0.36 \mathrm{~kg} / \mathrm{s}$ and operated at the peak efficiency point [22].

According to the setup of the particle feeder and the compressor operation, the contaminant concentration used in the present work allows the acceleration of the fouling process, reducing the exposure time during the lab-scale test by several orders of magnitude to the actual exposure time experienced by a land-based power unit. This technique is adopted mainly for studying the deposition phenomenon of cold and hot gas turbine sections [2 -4]. For this reason, the exposure time adopted in the present investigation is 30 min that covers about 500 operating hours of an actual installation [8].

\subsection{Experimental procedure}

The entire experimental procedure is a result of several steps, defined for ensuring the repeatability of the fouling assessment. The following steps are carried out for testing the compressor unit at $80 \% \mathrm{RH}$, and for a rotational speed of $20,000 \mathrm{rpm}$ :

- stator case disassembling and the picture of the clean stator and rotor surfaces are taken;

- compressor run for reaching the stable thermal condition (air temperature value at the compressor outlet) and the proper relative humidity value at the inlet $(80 \% \mathrm{RH})$;

- acquisition of the performance in clean condition;

- compressor fouling: $30 \mathrm{~min}$ of compressor operation at 20,000 rpm (corrected rotational speed) with constant particles ingestion (336 million particles per second) and acquisition of the performance degradation over time (the data acquisition is performed every $2 \mathrm{~min}$ ) related to the pressure ratio provides by the unit;

- stator case disassembling and the picture of the fouled stator and rotor surfaces are taken.
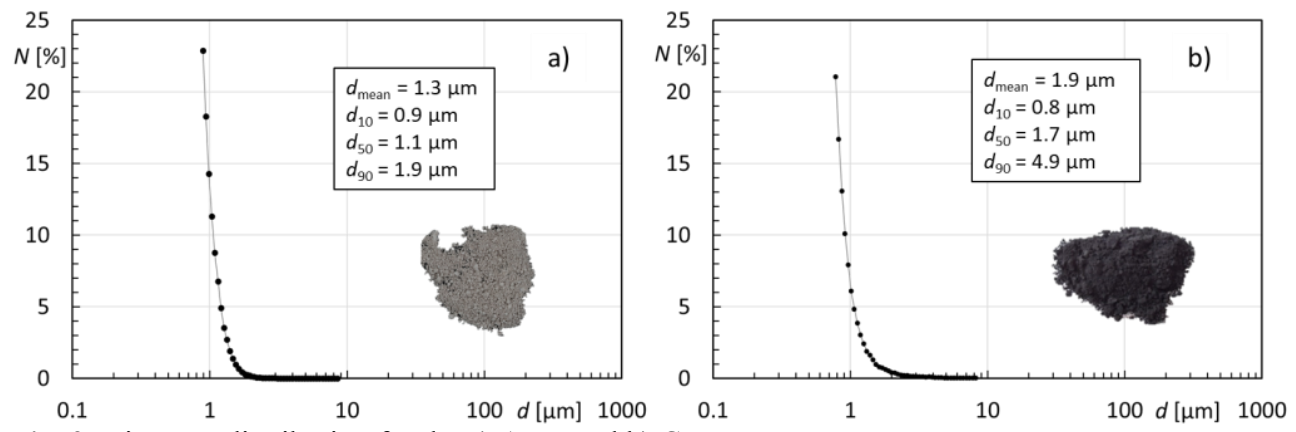

Fig. 2. Diameter distribution for the a) ARD and b) $C B$ 


\section{Results}

The experimental assessment of the fouling phenomenon has been carried out comprising three different approaches: compressor performance, particle adhesion capability, and deposit pattern detections.

\subsection{Performance}

The measurement of the compressor performance over the exposure time is based on the estimation of the pressure ratio across the axial stages (see Eq. 1). Figure 3a reports the overtime performance taken over the tests: in the $\mathrm{x}$-axis, the normalized duration of the test is reported while in the y-axis the ratio between the pressure ratio measured in clean and fouled conditions is reported. The pressure ratio of the clean condition is the value measured at the beginning of the test to compare the result (clean and fouled) for the same compressor setup (as reported, the compressor was disassembled after each test). For the sake of completeness, the compressor performance was taken under the clean and fouled condition for the same duration ( $30 \mathrm{~min}$ ) to assess the intrinsic variation (oscillation) of the compressor performance over time and increasing the statistical set of data. For increasing the readability, each trend is interpolated employing a straight line: the clean one is horizontal because, no performance loss was detected, while the two fouled trends are interpolated with a negative-slope line. The performance detection is carried out with an interval of $2 \mathrm{~min}$ and each dot is equipped with the uncertainty bar of $0.019 \%$ (see the uncertainty analysis proposed in the previous section).

Looking at the data, the pressure ratio values appear randomly dispersed and very similar to the data reported in the literature related to on-field detections $[7,8]$. The linear trend used for interpolating the data is adopted according to the literature [13] and allows the definition of the compressor performance drop. In particular, through these data, the ratio between the percentage losses in terms of the compression ratio per operating hour can be calculated. As can be seen in the plot, the contamination carried out with ARD powder appear more detrimental, and it determines a reduction of the pressure ratio over time of $0.48 \%$ per operating hour. In a similar way, but with fewer effects, the contamination with CB determines a reduction of $0.23 \%$ per operating hour. Such data relates the sensitivity of the compressor unit to the type of contaminant for a given operating condition $\left(22{ }^{\circ} \mathrm{C}\right.$ with $80 \% \mathrm{RH}$ ). Similar tests carried out at $22{ }^{\circ} \mathrm{C}$ with $50 \% \mathrm{RH}$, not reported here, have shown a reduction of $0.34 \% / \mathrm{h}$ and $0.09 \% / \mathrm{h}$ for $\mathrm{ARD}$ and $\mathrm{CB}$, respectively. The presence of high values of relative humidity increases the fouling effects, dramatically. Comparing the present results with the literature data [23], a gas turbine unit reduced its capacity by 350 BHP over 30 operating hours, which corresponds to a relative reduction of $0.5 \% / \mathrm{h}$, in line with the lab-test scaled results.
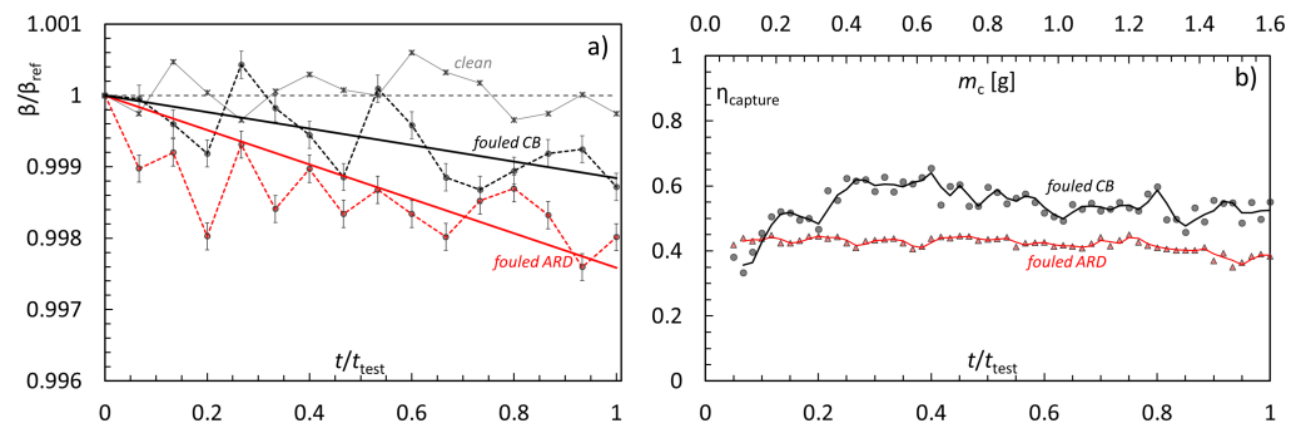

Fig. 3. Over time fouling effects: a) performance comparison of clean and fouled (ARD and CB) conditions and $\mathrm{b}$ ) capture trends over the exposure time by ARD and $\mathrm{CB}$ 
The assessment of the fouling effects for a multistage compressor unit is not straightforward. Effects on the performance due to fouling are a combination of the contemporary variation of pressure and flow coefficients. The presence of fouled parts over the compressor flow path could determine the reduction of the flow coefficient (reduction of the flow passage area and contemporary increment of the boundary layer thickness due to the increased surface roughness), which reflects on the increment of the pressure coefficient [12]. At the same time, the pressure coefficient could reduce by the action of internal losses generated by the modification of the aerodynamic geometry (e.g., the thickness of the leading edge). For these reasons, fouling acts stage-by-stage in a different way (each stage experiences different contamination due to flow conditions), introducing several non-linear effects, difficult-to-be-predicted. These non-linear effects, as well as the detachment process, could affect the slope of the performance trend. The linear interpolation of the performance is adopted based on data coming from actual detection, in which, in order to slow down the effects of fouling, a weekly online washing is usually carried out [24]. This means that, in standard applications, the fouling process have not sufficient time to develop, and in turn, the compressor unit has not the possibility to reach a sort of stable operating condition. In the light of these considerations, in [25] a different approach is proposed to interpret the performance trends based on non-linear behaviour.

\subsection{Sticking data}

The measurement of the compressor performance has been performed together with the measurement of the particle concentration at the compressor outlet section. The particle concertation has been measured by an online spectrometer able to sample the airflow contamination, continuously. Therefore, taking into account the particle flow rate injected at the compressor inlet (known and constant over the test) and the instantaneous value of the outflow particle concentration, the estimation of the deposited particle inside the compressor flow path is easily assessed. Figure $3 \mathrm{~b}$ shows the capture efficiency (defined as the ratio between the difference between the injected and discharged particles and the number of particles injected at the compressor inlet) overt the exposure time. Besides, the plot reports also the indication of the mass of contaminants that was ingested by the compressor to facilitate a scaling process of the present data based on the contaminant mass. As can be seen from the depicted data, the first part (1 min) of the particle sampling (and thus the consequent capture efficiency) was neglected due to the inaccuracy generated by the transient phenomena at the beginning of the test: the compressor internal volume is entirely free of particles, and the spectrometer operates in a clean airflow stream measuring a particle concentration lower than the actual value. The trends used for interpolating the instantaneous data refer to a moving average based on $1 \mathrm{~min}$ of sampling. This means that the average process is weighted using two samples.

Looking at the data, the capture efficiency appears almost constant over the exposure time for both tests. This means that the fouling process develops according to a linear trend and the sticking conditions remain almost constant over this investigation windows. A slight reduction of the capture efficiency is identified for an exposure time higher than 15 min (equal to 0.5 in the $\mathrm{x}$-axis). The $\mathrm{CB}$ contaminant appears stickier that ARD probably due to the interaction between humidity, blade surface, and the material of the micro-sized particles (the dimension of the $\mathrm{CB}$ particles is very similar to those characterized the ARD particles, see Fig. 2). In addition, the CB capture efficiency trend appears less stable over time and this could be due to a higher propensity of this powder to detach from the internal surfaces, increasing the concentration at the compressor outlet instantaneously.

Considering the fouling issue, non-linear effects are induced by local impact condition (in terms of particle sticking) and to the different fouling sensitivity of compressor units compared to the contaminant characteristics (particle diameter and powder nature). Both 
performance and capture efficiency are proposed in terms of the overall assessment of an actual compressor unit, but only with a detailed analysis of the deposit patterns, it is possible to complete the overall assessment of the fouling phenomenon.

\subsection{Deposit patterns}

In the last part of the present work, the analysis of the deposit patterns is reported. As reported in detail in the first part of this work, the detections of the flow path have been carried out with a fixed camera and light setup. Besides, the pictures have been postprocessed and compared employing the open-source software IMAGE J. The results of these operations are reported in Figs 4, 5 for the rotor blades and stator vanes, respectively. The pattern post-process is made up of the detection of the clean and fouled condition and the successive post-process. The greyscale pattern represents the pixel-by-pixel difference between the picture taken before and after the deposition test, and in turn, represents the fouled region of the compressor flow path. This means that the post-processed picture represents the compressor contamination after $30 \mathrm{~min}$ of exposure time.

The first finding related to these detections is related to the contamination over the stages. As reported, the contamination appears distributed over the entire compressor flow path, and even the rear stages are affected by particle deposition. The micro-sized dimension of the powders determines the capability of the particle to penetrate through the stages involving the entire blade surface. Therefore, no-specific actions provided by the centrifugal force has been noticed. Looking at the compressor rotor blades (Fig. 4) the deposit patterns show more significant contamination provided by $\mathrm{CB}$ (this is in line with the capture efficiency data early reported) that involves the entire pressure side of the six axial stages. The ARD deposits appear localized in the leading edge of the blades and at the blade tip and it seems to be also involved the trailing edge of the suction side. As described in the literature $[26,27]$, the deposits have a different role in performance degradation depending on the blade region involved. Moving to the analysis of the stator vanes, the deposit patterns are quite different. The CB powder affects the leading edge of the vanes and the blade tip, especially for the pressure side surface. The suction side is affected by deposits at leading and trailing edges, especially in the last two stages. The contamination provided by the ARD powder seems to be more uniform on the vanes without specific darker regions over the stages. From the visual inspection of the unit, no erosion and abrasion issues were discovered over the tests.

The present strategy adopted for the image post-processing allows pushing forward the fouling analysis, from a qualitative to a quantitative point of view. Taking as reference the post-processed images reported in Fig. 5, and by counting the grey and black pixels for each vane surface, it is possible to calculate the ratio between the number of the grey and black pixels (dirty regions on the vane surface) and the total number of the pixel on the vane surface. This value quantifies the fouled portion (in terms of covered area) of the vane surface. Figure 6 reports the comparison among the pixel ratio (pxr) values obtained for the two studied cases, divided according to the pressure (continuous lines) and suction (dashed lines) side of the vane surface. The $\mathrm{CB}$ powder seems to more detrimental for the pressure side, reaching $70 \%$ of coverage, while ARD powder can cover both pressure and suction side, similarly.

This quantitative information based on the image analysis tool could be useful for estimating the fouling intensity, for classifying the severity of the contamination, as a function of the contaminant type and operating conditions. Besides, the removal capability of the washing process could be addressed as well as the extraction of particle deposition information useful for developing and validating numerical simulation tools [28, 29]. 

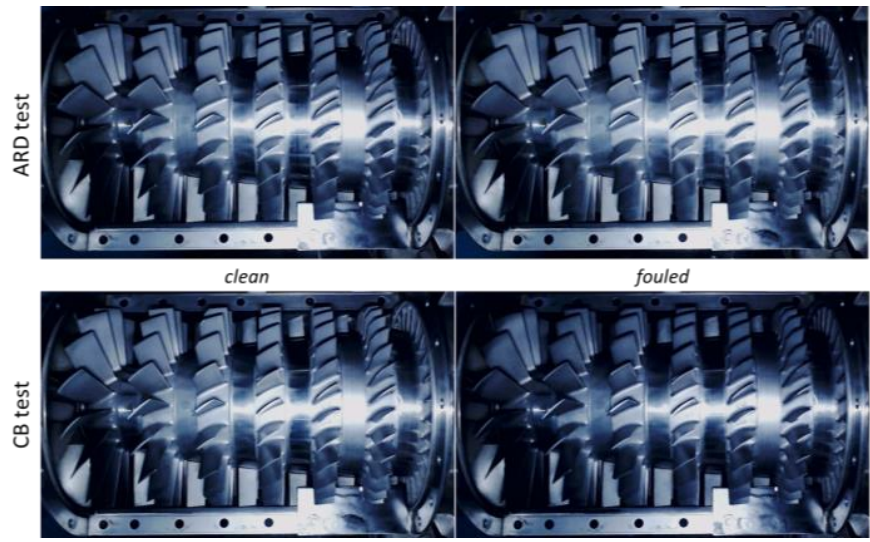

post-processed

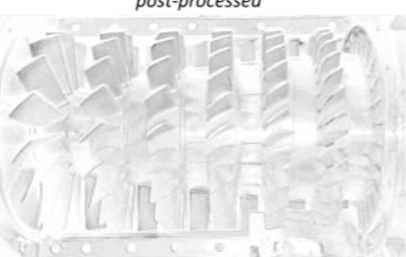

Fig. 4. Detections of the rotor blades deposits: clean, fouled (by ARD and CB powders) and postprocessed image
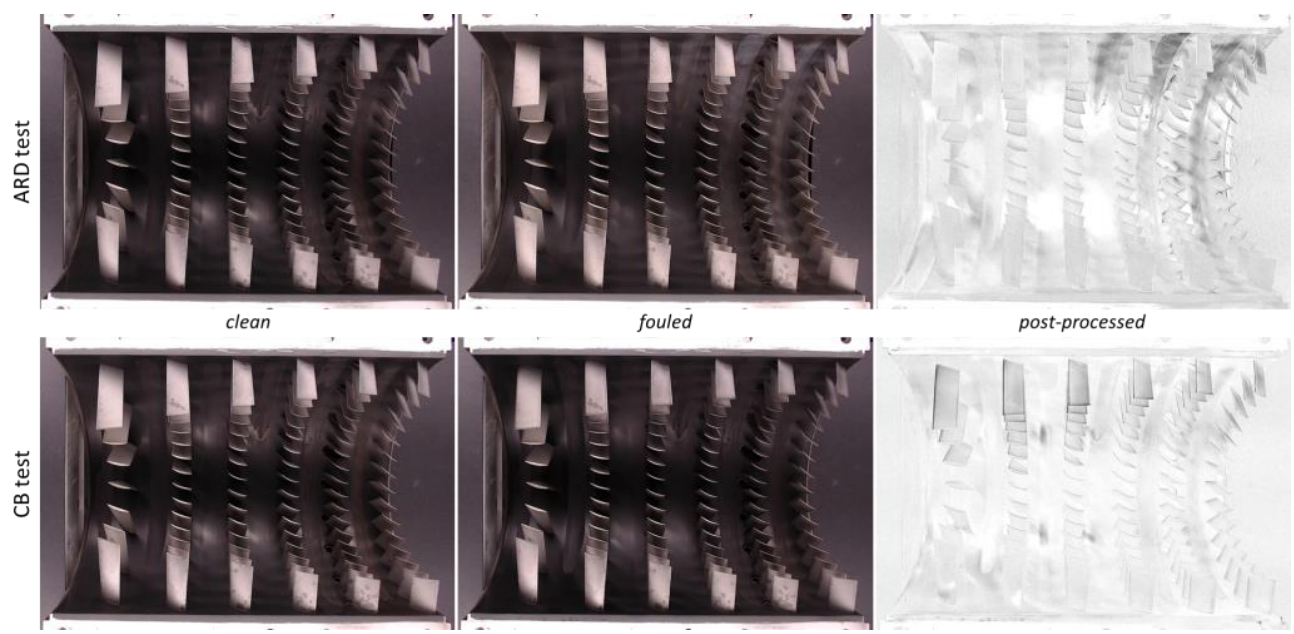

Fig. 5. Detections of the stator vanes deposits: clean, fouled (by ARD and CB powders) and postprocessed image

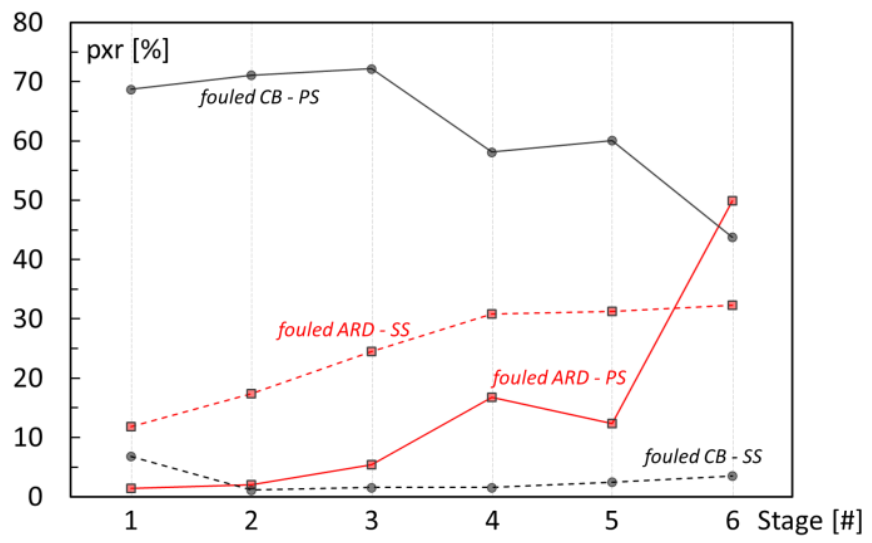

Fig. 6. Pixels ratio obtained from the stator vanes image analysis 


\section{Conclusions}

In this work, a comprehensive assessment of the fouling issue related to a multistage axial compressor has been reported. The experimental setup and the compressor contamination operations have been designed for tackling the fouling issue by three fronts: unit performance, particle sticking, and deposit pattern. The contamination of a multistage axial unit has been performed using two different contaminants for discovering the effects of soil and soot in the presence of high relative humidity value. Over the exposure time, the contaminant concentration has been kept constant and higher than that characterizes a common application, and the compressor performance and the particle counts have been measured repeatedly.

The performance analysis has shown that the degradation of the pressure ratio reaches the value of $0.5 \%$ per operating hour, and it is in the same order of magnitude for soil and soot contaminants. Looking at the sticking data, soot particles seem to be stickier than the soil and an almost constant trend of the overall capture efficiency of the compressor has been highlighted. State this, it is necessary to couple the information related to performance with the capture efficiency for depicting the fouling phenomenon entirely. Aiming by this, the detection of the deposits has been performed over the compressor flow path. Image analysis shows the different preferable zone on which soil and soot stick to the compressor internal surfaces. Pressure and suction surfaces, as well as the leading and trailing edges, show different contamination as a function of contaminant type and according to the stage.

The assessment of the fouling phenomenon should comprise several aspects related to particle and compressor unit behaviour. The combination of deposition pattern, performance degradation, and sticking properties is far from linear, introducing several complexities in the prediction of the development of fouling and at the same in the definition of general guidelines to mitigate and/or eliminate every issue. Lab-scaled tests allow the proper control of each variable related to one of the phenomena mentioned above, representing a good way for reducing the fouling effects on performance and increasing the machine reliability.

\section{References}

1. R. Kurz and K. Brun, J Eng. Gas Turb. Power, 134, 032401 (2012)

2. A. Suman, N. Casari, E. Fabbri, L. di Mare, F. Montomoli and M. Pinelli, Prog. Ener. Comb., 74, 103-151 (2019)

3. A. Suman, N. Casari, E. Fabbri, M. Pinelli, L. di Mare and F. Montomoli, J Eng. Gas Turb. Power. 141, 032601 (2019)

4. A. Suman, M. Morini, N. Aldi, N. Casari, M. Pinelli and P. R. Spina, J Turbomach. 139, 041005 (2017)

5. A. D. Mezheritsky and A. V. Sudarev, The mechanism of fouling and the cleaning technique in application to flow parts of the power generation plant compressors, 90-GT103, Proceedings of the ASME Turbo Expo, edit by ASME, NY (1990)

6. J.-P. Stalder, J Eng. Gas Turb. Power, 23, 363-370 (2001)

7. I. S. Diakunchak, J Eng. Gas Turb. Power, 114, 161-168 (1991)

8. C. A: Perullo, T. Lieuwen, J. Barron, D. Grace and L. Angello, Evaluation of air filtration options for an industrial gas turbine, GT2015-43736, Proceedings of the ASME Turbo Expo, edit by ASME, NY (2015)

9. C. B: Meher-Homji, M. Chaker and A. F. Bromley, The fouling of axial flow compressors - Causes, effects, susceptibility and sensitivity, GT2009-59239, Proceedings of the ASME Turbo Expo, edit by ASME, NY (2009) 
10. A. Silingardi, P. Astrua, S. Piola, and I. Ventrucci, A Method for a Reliable Prediction of Heavy Duty Gas Turbines Performance Degradation due to Compressor Aging Employing Field Test Data, Proceedings of Power Gen Europe, June 4-6, 2013, Messe, Wien, Austria (2013)

11. A. P. Tarabrin, V. A. Schurovsky, A. I. Bodrov and J.-P. Stalder, Influence of axial compressor fouling on gas turbine unit performance based on different schemes and with different initial parameters, 98-GT-416, Proceedings of the ASME Turbo Expo, edit by ASME, NY (1998)

12. F. Melino, A. Peretto and P. R. Spina, Development and validation of a model for axial compressor fouling simulation, GT2010-22947, Proceedings of the ASME Turbo Expo, edit by ASME, NY (2010)

13. G. F. Aker and H. I. H. Saravanamuttoo, J Eng. Gas Turb. Power, 111, 343-350 (1989)

14. T. Zaba and P. Lombardi, Experience in the Operation of Air Filters in Gas Turbine Installations, 84-GT-39, Proceedings of the ASME Turbo Expo, edit by ASME, NY (1984) 15. Allison Gas Turbine. Operation and Maintenance manual. Turboshaft models 250C18, A, B \& C. 15 September 1971, Rev. 16 June 1975. Detroit Diesel Allison, Division of General Motors Corporation, Indianapolis, IN 46206

16. W. C. Hinds. Aerosol technology: properties, behavior, and measurement of airborne particles (Wiley \& Sons, US, 1999)

17. Standard ISO/IEC GUIDE 98-3:2008. Uncertainty of measurement - Part 3: Guide to the expression of uncertainty in measurement (GUM:1995)

18. JCGM 100:2008. Evaluation of measurement data - Guide to the expression of uncertainty in measurement

19. C. A. Schneider, W. S. Rasband, and K. W: Eliceiri, Nat. Methods, 9, 671 (2012)

20. K. Brun, T. A. Grimley, W. C. Foiles and R. Kurz, J Eng. Gas Turb. Power, 137, 042605 (2015)

21. Standard ISO 12103-1:2016. Road vehicles - Test contaminants for filter evaluation Arizona test dust

22. E. Munari, M. Morini, M. Pinelli, P. R. Spina and A. Suman, J Eng. Gas Turb. Power, 139, 022605 (2017)

23. F. R. Harris, A 7500-SHP Gas Turbine for Naval Boost Propulsion, 61-GTP-5, Proceedings of the ASME Turbo Expo, edit by ASME, NY (1961)

24. E. Schneider, S. D. Bussjaeger, S. Franco, D. Therkorn, J Eng. Gas Turb. Power 132, 062001 (2010)

25. A. Vulpio, A. Suman, N. casari, M. Pinelli, R. Kurz, K. Brun, Analysis of time-wise compressor fouling phenomenon on a multistage test compressor: performance losses and particle adhesion, GT2020-15418, Proceedings of the ASME Turbo Expo, edit by ASME, NY (2020)

26. N. Aldi, M. Morini, M. Pinelli, P. R. Spina, A. Suman and M. Venturini, J Turbomach. 136, 021016 (2013)

27. M. Morini, M. Pinelli, P. R. Spina and M. Venturini, J Eng. Gas Turb. Power 133, 072402 (2011)

28. A. Suman, A. Vulpio, N. Casari, M. Pinelli, K. Rainer, K. Brun, Deposition pattern analysis on a fouled multistage test compressor, GT2020-15476, Proceedings of the ASME Turbo Expo, edit by ASME, NY (2020)

29. N. casari, M. Pinelli, P. R. Spina, A. Suman, A. Vulpio, Performance degradation due to fouling and recovery after washing in a multistage test compressor, GT2020-15488, Proceedings of the ASME Turbo Expo, edit by ASME, NY (2020) 